\title{
Wolbachia Are Present in Southern African Scorpions and Cluster with Supergroup F
}

\author{
Laura Baldo - Lorenzo Prendini - Angelique Corthals • \\ John H. Werren
}

Received: 6 December 2006/Accepted: 12 June 2007/Published online: 5 August 2007

(C) Springer Science+Business Media, LLC 2007

\begin{abstract}
The presence and distribution of the intracellular bacteria Wolbachia in the arthropod subphylum Chelicerata (including class Arachnida) has not been extensively explored. Here we report the discovery of Wolbachia in scorpions. Five strains found in host species of the genus Opistophthalmus (Southern African burrowing scorpions) have been characterized by Multilocus Sequence Typing and by Wolbachia Surface Protein. Phylogenetic analyses indicate clustering in the supergroup $\mathrm{F}$ and a high genetic relatedness among all scorpion strains as a result of a potential transmission within the host genus. The F-group is an uncommon lineage compared to the A and $\mathrm{B}$ supergroups, although it is present in a broad range of hosts (including insects, filarial nematodes, and now arachnids) and across a large geographical area (e.g., North America, Africa, Europe, and Australia). It also shows no evidence of recombination and has a significantly higher genetic diversity than supergroup A and B. Overall, this pattern suggests an older radiation of F-strains with respect to A and B-strains, followed by limited horizontal
\end{abstract}

L. Baldo $(\bowtie)$

Department of Biology, University of California,

900 University Avenue, Riverside, CA 92521, USA

e-mail: laurab@ucr.edu

\section{Prendini}

Division of Invertebrate Zoology, American Museum of Natural History, Central Park West at 79th Street, New York, NY 10024-5192, USA

\footnotetext{
A. Corthals

Faculty of Life Sciences, University of Manchester, Jackson's Mill, Sackville Street, M60 1QD Manchester, UK

J. H. Werren

Department of Biology, University of Rochester, Rochester, NY 14627-0211, USA
}

transmission across host genera and reduced genetic flux among strains. A more extensive sampling of supergroup F-strains is required to confirm this scenario.

Keywords Wolbachia - Endosymbiont - MLST . Opistophthalmus

\section{Introduction}

The intracellular bacteria Wolbachia pipientis are present in a broad range of hosts, having to date been found in filarial nematodes and all major arthropod taxonomic groups, including insects, terrestrial crustaceans, and chelicerates [8, 22, 28]. The distribution of Wolbachia not only covers a large taxonomic diversity of host species, but also an extensive geographical area; these bacteria are found on all major continents, except Antarctica.

The ability of Wolbachia to manipulate the cell biology and reproduction of hosts has attracted the attention of biologists [27]. Manipulations including parthenogenetic induction, feminization, male-killing and cytoplasmic incompatibility are known to have a dramatic effect on the genetic structure and history of arthropod populations [25].

Wolbachia contains a remarkable genetic diversity that has allowed classification of the strains into eight supergroups $(\mathrm{A}-\mathrm{H})[5,6,22]$, although decommissioning of supergroup $\mathrm{G}$ has been recently proposed for insufficient and inconsistent data [4]. Except for supergroups C and D, specific to filarial nematodes, the remaining supergroup strains are found in a broad spectrum of arthropod host species. The full range of hosts and genetic diversity of Wolbachia has not yet been fully explored, although new phylogenetic lineages and hosts are increasingly discovered [6]. Among Chelicerata, Wolbachia infection was recently reported in spiders, 
mites, ticks, and one species of pseudoscorpion [11, 12, 22, 29]. No report of Wolbachia infection exists for scorpions.

Here we show that Wolbachia infections occur in scorpions. Wolbachia were found in several species of the scorpion genus Opistophthalmus (family Scorpionidae), commonly known as the southern African burrowing scorpions. Opistophthalmus is a radiation comprising more than 59 species, the majority of which are endemic to Namibia and South Africa [19, 21]. Recently, a Multilocus Sequence Typing (MLST) scheme was developed for Wolbachia (based on five loci, gatB, $\operatorname{cox} A, h c p A, f t s Z$, and $f b p A$ ), creating the basis for accurate characterization of strains, and offering an expanding dataset for integrative studies of the genetics, geographical distribution, and host range of these common bacteria (http://www.pubmlst.org/wolbachia/) [2]. The Wolbachia surface protein (WSP) is used as an additional marker of strain variability. Although the function of WSP remains unknown, the four hypervariable regions (HVRs) of the protein are subject to extensive recombination and likely are involved in the host-symbiont interaction [3]. A typing system based on the profile of the four HVR peptides of a WSP sequence was recently developed [2].

Here we report characterization by MLST and WSP of Wolbachia strains found in five species of Opistophthalmus and discuss the genetic relatedness and history of these strains in the scorpions. This establishes that Wolbachia infections occur in scorpions, and provides a first example of use of MLST for survey of Wolbachia strain variability in closely related host species.

\section{Materials and Methods}

Samples, PCR, and Sequencing

Twelve specimens, representing seven described and four undescribed species of the scorpion genus Opistophthalmus
(Table 1), were screened for Wolbachia infection using 16S rRNA Wolbachia-specific primers and standard polymerase chain reaction (PCR) protocols [16]. The specimens, collected between 1997 and 1998, are presently stored in the Ambrose Monell Cryo Collection (AMCC) at the American Museum of Natural History. They were originally fixed and stored in $96 \%$ ethanol at $-20^{\circ} \mathrm{C}$, subsequently transferred to pregenerated barcoded cryo-vials, using sterile instruments, and contained in a biosafety cabinet immersed in the liquid nitrogen vapor phase cryogenic vats (at $-160^{\circ} \mathrm{C}$ ). The Australian termite Coptotermes acinaciformes, infected with supergroup FWolbachia [6], was also included in the study for phylogenetic analyses. DNA was extracted from the gonads of single scorpion specimens and from the whole termite using the DNAeasy Tissue Kit (Qiagen, Germantown, MD). MLST and wsp gene sequences were amplified using standard primers and PCR protocols (available at http://www.pubmlst.org/wolbachia/) [2]. PCR products were purified using Montage PCR centrifugal Filter Devices (Millipore) and sequenced in both directions. Sequences were deposited at the MLST website. All samples were screened using nematode-specific $18 \mathrm{~S}$ primers [10] to exclude contamination with parasitic nematode DNA (and potential associated Wolbachia symbionts). Results were negative.

\section{Genetic Diversity of Wolbachia}

Estimates of genetic diversity (Pi), variable sites (VI), and ratio of synonymous substitutions per synonymous site over nonsynonymous substitutions per nonsynonymous site $\left(K_{a} / K_{s}\right)$ were performed with DNAsp vs 4.10.2 [23]. Recombination analyses were conducted on single and concatenated MLST gene alignments of supergroup

Table 1 Opistophthalmus species positive for Wolbachia infection

\begin{tabular}{|c|c|c|c|c|c|c|}
\hline Species & Specimen (AMCC \#) & Country & Province & District & Latitude & Longitude \\
\hline O. ammopus Lamoral, 1980 & 100798 & South Africa & Northern Cape & Namaqualand & $29^{\circ} 17^{\prime} \mathrm{S}$ & $17^{\circ} 05^{\prime} \mathrm{E}$ \\
\hline O. capensis Herbst, 1800 & 100812 & South Africa & Western Cape & Hopefield & $33^{\circ} 08^{\prime} \mathrm{S}$ & $18^{\circ} 00^{\prime} \mathrm{E}$ \\
\hline O. chaperi Simon, 1880 & 100818 & South Africa & Western Cape & Worcester & $33^{\circ} 39^{\prime} \mathrm{S}$ & $19^{\circ} 32^{\prime} \mathrm{E}$ \\
\hline O. granifrons Pocock, 1896 & 100840 & South Africa & Northern Cape & Namaqualand & $30^{\circ} 19^{\prime} \mathrm{S}$ & $18^{\circ} 15^{\prime} \mathrm{E}$ \\
\hline O. latimanus Koch, 1841 & 100860 & South Africa & Eastern Cape & Uitenhage & $33^{\circ} 43^{\prime} \mathrm{S}$ & $25^{\circ} 17^{\prime} \mathrm{E}$ \\
\hline O. latro Thorell, 1876 & 100862 & South Africa & Western Cape & Hopefield & $33^{\circ} 08^{\prime} \mathrm{S}$ & $17^{\circ} 59^{\prime} \mathrm{E}$ \\
\hline O. litoralis Lawrence, 1955 & 100869 & Namibia & Kunene Region & Opuwo & $19^{\circ} 22^{\prime} \mathrm{S}$ & $12^{\circ} 42^{\prime} \mathrm{E}$ \\
\hline Opistophthalmus sp. 1 & 100921 & South Africa & Northern Cape & Namaqualand & $28^{\circ} 37^{\prime} \mathrm{S}$ & $16^{\circ} 52^{\prime} \mathrm{E}$ \\
\hline Opistophthalmus sp. 2 & 100922 & South Africa & Western Cape & Ceres & $32^{\circ} 51^{\prime} \mathrm{S}$ & $19^{\circ} 17^{\prime} \mathrm{E}$ \\
\hline Opistophthalmus sp. 3 & 100925 & South Africa & Northern Cape & Namaqualand & $29^{\circ} 22^{\prime} \mathrm{S}$ & $17^{\circ} 40^{\prime} \mathrm{E}$ \\
\hline Opistophthalmus sp. 3 & 100926 & South Africa & Northern Cape & Namaqualand & $29^{\circ} 22^{\prime} \mathrm{S}$ & $17^{\circ} 40^{\prime} \mathrm{E}$ \\
\hline Opistophthalmus sp. 4 & 100928 & South Africa & Northern Cape & Namaqualand & $29^{\circ} 22^{\prime} \mathrm{S}$ & $18^{\circ} 53^{\prime} \mathrm{E}$ \\
\hline
\end{tabular}


F-sequences using the MaxChi method, implemented in RDP2 program [14]. Parameters were set as follows: triplets were scanned using different values of fraction of variable sites per window, a Bonferroni correction applied, and 1000 permutations generated. The highest acceptable $P$ value cut-off was set to 0.05 . Statistical significance of difference in synonymous divergence within supergroups was calculated using the MannWhitney test based on $K_{s}$ values of pairwise comparisons of $f t s Z$ sequences for each supergroup with respect to the other supergroups.

\section{Phylogenetic Analyses}

Maximum likelihood (ML) and maximum parsimony (MP) analyses were performed on the concatenated MLST and $f t s Z$ alignments using PAUP v 4.01 [26]. Modeltest v 3.06 [18] and the Akaike information criterion were used to select a model of substitution: GTR+I for MLST and $\mathrm{TrN}+\mathrm{I}+\mathrm{G}$ for ftsZ. Heuristic searches were conducted with tree-bisection-reconnection (TBR) branch-swapping and 10 replications of random stepwise addition. The same search settings were used for both likelihood and parsimony bootstrap analyses with 100 and 1000 replications respectively.

\section{Results}

All Opistophthalmus specimens screened for Wolbachia infection were positive based on 16S rRNA amplification. At least one MLST gene was sequenced for seven specimens (Table 2). Each infected specimen carried a single strain based on chromatogram analysis.
Scorpion STs Are Genetically Closely Related

All alleles and allelic profiles (i.e., Sequence Type, ST) characterized for Opistophthalmus strains were new to the MLST database (Table 2). Several alleles are shared among the profiles, indicating relatedness among strains. Five strains were fully typed by MLST and assigned an ST. Divergence among the five STs accounts for only 38 variable sites (VI) out of 2079 sites ( $\mathrm{Pi}=0.88 \%$ ). The gene $f b p A$ showed the highest nucleotide divergence per site $(1.6 \%)$, consistent with previous findings [2]. Strains have evolved mainly by synonymous substitutions as shown by average values of $K_{a} / K_{s}$ per gene $\ll 1$ (average $K_{a} / K_{s}$ across genes is 0.39 ). This is compatible with a scenario of strong purifying selection.

MaxChi analyses indicate no recombination events among the five STs based on either single gene or concatenated MLST data set alignments $(P<0.05)$.

\section{Scorpion Wolbachia Strains Cluster in Supergroup F}

Both ML and MP phylogenetic reconstructions based on the concatenated alignment of the five MLST genes indicate a strong clustering of all five scorpion STs with supergroup F, together with F-strains from the bed bug, Cimex lectularius, and the termite, C. acinaciformes (Fig. 1) (likelihood and parsimony bootstrap values, $P=$ 100). Analysis of the relationships among scorpion strains supports two main clusters: (O. chaperi, O. granifrons), $(O$. capensis, O. litoralis, O. latimanus).

\section{Scorpion Strains Are Remarkably Divergent at WSP}

Three of the five strains fully typed by MLST were also genotyped based on WSP (Table 2). The three $w s p$ alleles

Table 2 Complete and partial MLST and WSP profiles of Wolbachia strains hosted in seven Opistophthalmus species

\begin{tabular}{|c|c|c|c|c|c|c|c|c|c|c|c|c|}
\hline \multirow[b]{2}{*}{ Strain ID ${ }^{\mathrm{a}}$} & \multirow[b]{2}{*}{ Host species } & \multirow[b]{2}{*}{$\mathrm{ST}^{\mathrm{b}}$} & \multicolumn{5}{|c|}{$\mathrm{MLST}^{\mathrm{c}}$} & \multirow[b]{2}{*}{$w s p^{\mathrm{d}}$} & \multicolumn{4}{|l|}{$\mathrm{WSP}^{\mathrm{e}}$} \\
\hline & & & gatB & $\operatorname{cox} A$ & hcpA & $f t s Z$ & $f b p A$ & & HVR1 & HVR2 & HVR3 & HVR4 \\
\hline 43 & O. capensis & 62 & 29 & 30 & 34 & 27 & 31 & 36 & 26 & 26 & 29 & 28 \\
\hline 44 & O. latimanus & 64 & 31 & 30 & 36 & 28 & 32 & 37 & 27 & 27 & 30 & 29 \\
\hline 42 & O. granifrons & 72 & 30 & 31 & 35 & 48 & 33 & 35 & 25 & 25 & 28 & 27 \\
\hline 59 & O. chaperi & 78 & 30 & 55 & 72 & 48 & 70 & I & l & l & l & l \\
\hline 45 & O. litoralis & 77 & 29 & 56 & 58 & 60 & 57 & I & l & I & l & I \\
\hline 60 & O. aтmориs & n.a. & 29 & I & I & 59 & 31 & l & l & I & l & I \\
\hline I & O. latro & n.a. & I & I & I & I & 70 & I & I & I & l & I \\
\hline
\end{tabular}

${ }^{\text {a }}$ Wolbachia strain identifier as assigned by the MLST database

b ST = Sequence Type, it identifies a unique allelic profile (assigned only to strains fully characterized by MLST)

c,d Numbers refer to nucleotide alleles. Alleles shared among STs are in italics

e Numbers refer to peptide haplotypes of the four consecutive sections of WSP, each including a hypervariable region (HVR)

Incomplete profiles are due to multiple failed PCRs 


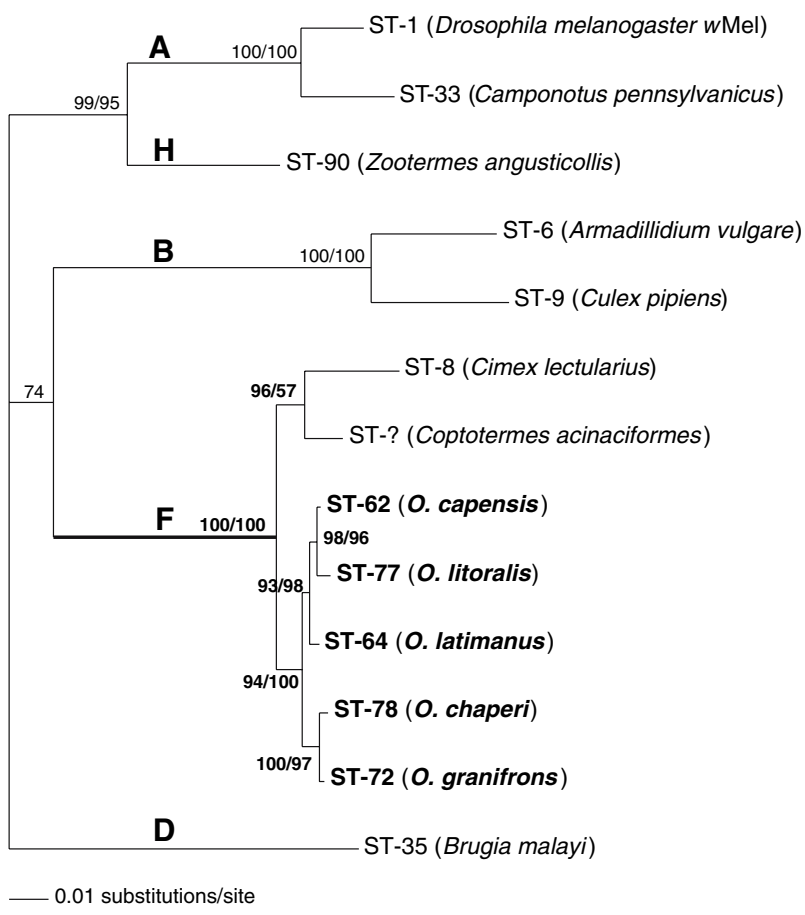

Fig. 1 Maximum likelihood (ML) tree of 13 Wolbachia STs, representative of five supergroups, based on the concatenated alignment of MLST loci (2079 bp). The five scorpion strains here typed are shown in bold. Because of absence of recombination among scorpion strains at the MLST genes, this phylogeny would represent actual genetic distances and relationships among these strains. In brackets are examples of host species harboring the Wolbachia ST (we note that the same ST can be found in diverse host species). The strain from $C$. acinaciformes was only partially typed by MLST (missing $\operatorname{coxA}$ allele) and thus not assigned an ST. ML and MP bootstrap values are shown at each node (left to right)

as well as HVR peptides were new to the database. Whereas the average divergence among the three strains at MLST genes is less than 1\% (26 out of $2079 \mathrm{VI}$ ), their wsp nucleotide sequences alone differ by $4.4 \%$ (excluding gaps), with 44 VI of 498 sites, including a 12-bp gap at allele 35 harbored by the $O$. granifrons strain (Fig. 2). A large fraction of these polymorphisms (19) occurs at HVR4 and are nonsynonymous $\left(K_{a} / K_{s}=1.66\right)$. To verify whether the divergence at HVR4 between scorpion strains was the result of recombination, we searched for potential donor sequences by performing a blastp search of the HVR4 peptides of the three scorpion WSP sequences (peptide \#27, 28, and 29, Table 2). The blastp results gave best matches for each HVR4 peptide with less than $75 \%$ of amino acid identity to the query sequence, suggesting that variation at HVR4 among scorpion sequences is either the result of high mutation rates at this region, or that a potential donor sequence has not yet been identified.

\section{Genetic Variability of Supergroup F}

We retrieved all F- $f t s Z$ alleles thus far available in the MLST and Genbank databases to explore the genetic variation among F-strains. The final dataset comprises 16-F strains (corresponding to 15 different alleles). ML phylogeny (Fig. 3) confirms clustering of all above strains in supergroup $\mathrm{F}$ (ML and MP bootstrap $\mathrm{P}=60$ and 90, respectively). Relationships within the group are only partially solved because of the high level of nucleotide conservation at this gene (mean $\mathrm{Pi}=2.7 \%$ ). Significant bootstrap support is given for all scorpion strains $(\mathrm{P}=73$ and 96), the two bush crickets ( $\mathrm{P}=90$ and 88$)$, and the two Australian Coptotermes termites $(P>50)$.

We also explored the genetic diversity of supergroup $\mathrm{F}$ with respect to supergroup A and B, by retrieving all $\mathrm{A}$ and B ftsZ sequences available at the MLST database at the time of this study. The final dataset included 19 A-strains (9 alleles), 17-B strains (14 alleles), and the 16-F strains (15 alleles).

Based on the $f t s Z$ alignment, the average synonymous divergence $\left(K_{s}\right)$ within supergroup $\mathrm{F}$ is $11.06 \%$, significantly greater than within supergroup A $(4.45 \%)$ and supergroup B (7.66\%) (Mann-Whitney test, $P<0.0001$ ). Scorpion sequences form a genetically distinct group from the rest of the F-dataset (mean nucleotide differences between the two datasets is 14.08).

Consistent with results based on scorpion STs alignment, analysis of recombination based on the ftsZ alignment failed to detect any recombination event within supergroup F (MaxChi, $P<0.05$ ).
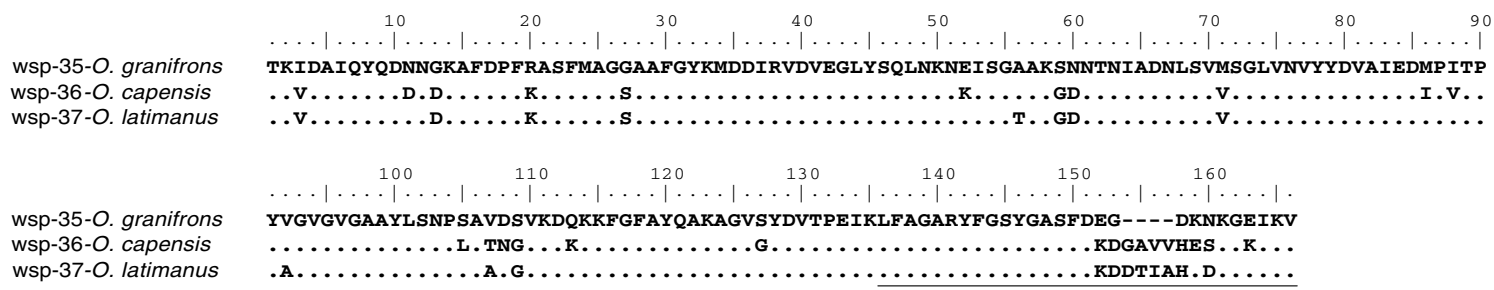

Fig. 2 Wolbachia surface protein (WSP) amino acid alignment of the three scorpion strains. Note the remarkable divergence at WSP compared to the Multilocus Sequence Typing divergence (Fig. 1). Amino acid range of HVR4 is underlined 


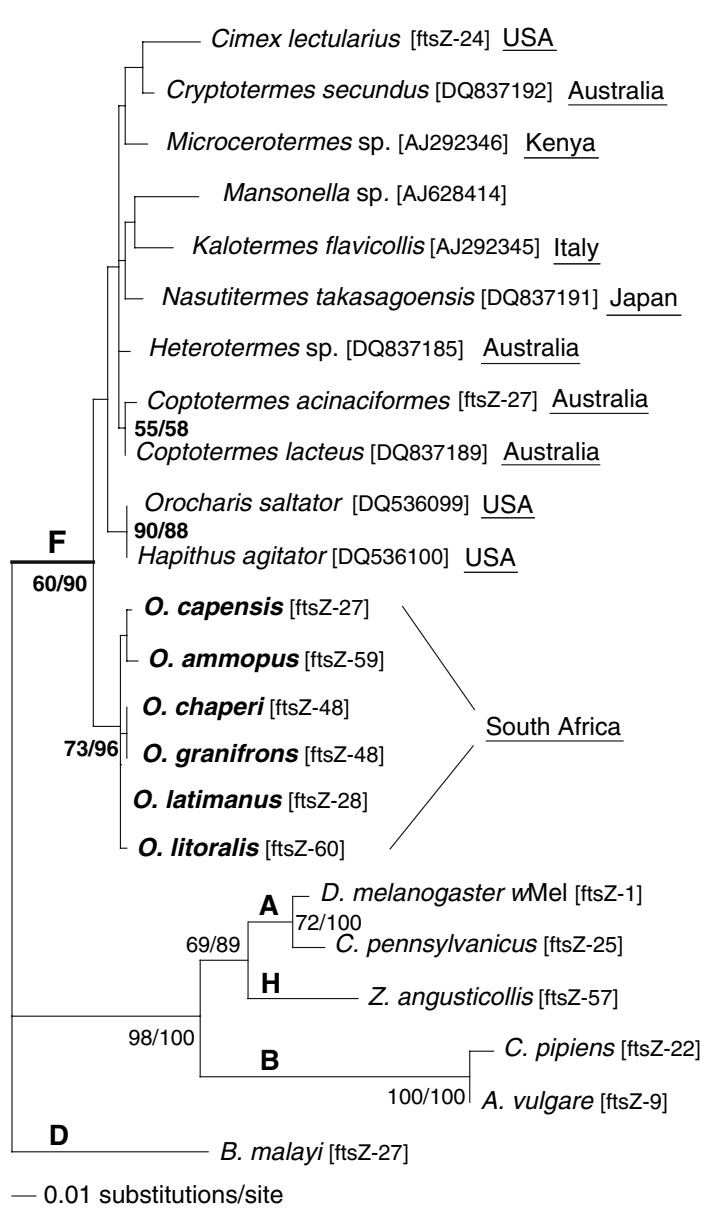

Fig. 3 Maximum likelihood (ML) tree based on the $f t s Z$ alignment (435 bp). The $\mathrm{F}$ dataset includes, in addition to strains in Fig. 1, sequences retrieved from Genbank or obtained in this study (i.e., Opistophthalmus ammopus). For each strain, the $f t s Z$ accession number or allele is given in brackets. Scorpion strains are boldfaced. ML and MP bootstrap values are shown at each node (left to right)

\section{Discussion}

This study represents the first report of Wolbachia in a major group of arthropods, the arachnid order Scorpiones. The scorpion Wolbachia strains found belong to supergroup F, a widespread (geographically and in host taxa) but relatively uncommon lineage of Wolbachia. Among the unanswered questions is whether F-Wolbachia occur commonly within scorpions, or if this is a unique feature of the genus Opistophthalmus.

\section{Scorpion Strains Are Monophyletic}

All Wolbachia strains characterized in the genus Opistophthalmus are genetically very closely related (less than $1 \%$ variation). Such relatedness of strains over a large geographical range, encompassing different localities in
South Africa and Namibia, but within the same host genus, indicates that the scorpion Wolbachia strains form a monophyletic group. Consistent with this hypothesis, STs and WSP sequences of the scorpion strains are more closely related to each other than to any other STs or WSPs thus far characterized. This finding could also reflect poor strain sampling within supergroup F, for which only one complete and one incomplete ST, in addition to the scorpion strains, are currently available (Fig. 1). Based on ftsZ, however, for which a larger subset of F-sequences is available, scorpion strains were nevertheless more closely related to each other than to any other F-strains.

Intraspecific Wolbachia Horizontal Transfer Versus Host-Symbiont Codivergence

Although Wolbachia strains in Opistophthalmus might have evolved within this host genus, as suggested by genetic data, this does not imply exclusive vertical transmission (codivergence) of Wolbachia from a common ancestor of the scorpion host genus. The alternative scenario would involve horizontal strain transfer among Opistophthalmus species. Radiation within this genus probably occurred within the last 5-10 Myr based on geological data [21]. Considering a rate of substitution for Wolbachia of $10^{-8}$ substitutions per synonymous site per year [7], the divergence time between the two most divergent scorpion STs (77 and 78, $K_{s}=2.84 \%$ ) is about $1.8 \mathrm{Myr}$. This result is compatible with monophyly of the scorpion Wolbachia clade. Reconstruction of species relationships within the genus Opistophthalmus is currently in progress by one of the authors, and preliminary data based on morphology, three mitochondrial and three nuclear gene loci, indicate the following relationships among the five species for which Wolbachia strains have been fully typed: (O. litoralis ((O. chaperi, O. latimanus) (O. granifrons, $O$. capensis)). Such relationships differ from Wolbachia MLST-based relationships (Fig. 1), and would exclude a strict host-symbiont codivergence, instead supporting a scenario of strain horizontal transfer among Opistophthalmus species.

The specimens analyzed here originate from localities broadly distributed within South Africa and Namibia (Table 1). These scorpion species are philopatric, habitatspecialists with very limited vagility [20], suggesting little opportunity for contact among them [21]. In a scenario where horizontal transfer of Wolbachia strains is facilitated by contact among host individuals, we might expect that species found in close regions harbor more closely related strains than distant species. The geographical distribution of the specimens studied here does not mirror such a pattern, however. Strains from species found at distant 
localities in Namibia and the Western Cape Province of South Africa ( $O$. litoralis and $O$. capensis, respectively) group together and share identical alleles, whereas strains from the same region (O. chaperi and $O$. capensis) do not (Table 2 and Fig. 1).

Opistophthalmus species are generalist predators, and they prey mostly on terrestrial arthropods (insects, myriapods, and arachnids, including other scorpions). Feeding on common prey, infected with Wolbachia, could provide the link for horizontal transfer of the same strain across related species.

Routes and vectors of Wolbachia horizontal transfer remain one of the main unsolved questions. It is, for example, unclear whether horizontal transfer of Wolbachia is more likely to occur within the same host taxonomic group (at different levels, such as genus or species, as suggested here for scorpions) than among different groups. Phylogenetic relatedness among strains within the same host genus has been documented in several studies, often on the basis of one or two genes, as for instance in Drosophila species [15], and would need to be revisited in light of the extensive recombination found in Wolbachia genomes [1, 2]. More MLST data from different host groups and different geographical regions will help to answer this question.

Genetic, Host, and Geographical Variation of Supergroup F

Wolbachia strains belonging to supergroup $\mathrm{F}$ have thus far been found in several species of termites [13], filarial nematodes of the genus Mansonella [6], two species of bush crickets [17], several species of cimicids [24], three species of lice, and one species of louse-fly [9]. In terms of geographical distribution, F-strains have thus far been found in host species from North America (U.S. states of AL, CA, MD, MS), South America (Venezuela), Europe (Italy), Africa (Kenya, Ethiopia, Namibia, and South Africa), Japan, Indonesia, and Australia. The pattern of strain diversity does not mirror the geographical distribution of the host species (Fig. 3). For instance, based on the fts $Z$ tree, the strain from the termite Microcerotermes sp. (Kenya) is more closely related to the termite $C$. acinaciformes (Australia) than to the southern African scorpions. Similarly, strains from Australia do not overall group, neither do strains from the United States.

Genetic data indicate that supergroup F strains are significantly more divergent than either supergroup A or B. This might be expected in a scenario of an older divergence among F-strains, and/or no recombination events within supergroup $\mathrm{F}$ and between $\mathrm{F}$ and other supergroups in the recent past. Consistent with these results, we did not detect any recombination within supergroup $\mathrm{F}$ based on either the concatenated MLST or ftsZ datasets. Furthermore, the relative paucity of F-strains compared to A- and B-strains supports a scenario of limited horizontal transfer (i.e., the major force of Wolbachia dispersion) of F-strains. Overall, the results suggest an older radiation of members of supergroup $\mathrm{F}$ with respect to both the supergroup $\mathrm{A}$ and $\mathrm{B}$ strains, followed by infrequent host shift across genera. A more extensive sampling of these F-strains and full MLST characterization will be necessary to confirm this scenario.

Acknowledgments We thank Arati Panda for her help in sample screening. This study was supported by U.S. National Science Foundation grant EF-0328363 to JHW.

\section{References}

1. Baldo L, Bordenstein S, Wernegreen JJ, Werren JH (2006) Widespread recombination throughout Wolbachia genomes. Mol Biol Evol 23:437-449

2. Baldo L, Dunning Hotopp JC, Jolley KA, Bordenstein SR, Biber SA, Choudhury RR, Hayashi C, Maiden MC, Tettelin H, Werren JH (2006) Multilocus sequence typing system for the endosymbiont Wolbachia pipientis. Appl Environ Microbiol 72:70987110

3. Baldo L, Lo N, Werren JH (2005) Mosaic nature of wsp (Wolbachia surface protein). J Bacteriol 187:5406-5418

4. Baldo L, Werren JH (2007) Revisiting Wolbachia supergroup typing based on WSP: spurious lineages and discordance with MLST. Curr Microbiol 55:81-87

5. Bordenstein S, Rosengaus RB (2005) Discovery of a novel Wolbachia super group in Isoptera. Curr Microbiol 51:393-398

6. Casiraghi M, Bordenstein SR, Baldo L, Lo N, Beninati T, Wernegreen JJ, Werren JH, Bandi C (2005) Phylogeny of Wolbachia pipientis based on gltA, groEL and ftsZ gene sequences: clustering of arthropod and nematode symbionts in the F supergroup, and evidence for further diversity in the Wolbachia tree. Microbiology 151:4015-4022

7. Clark MA, Moran NA, Baumann P (1999) Sequence evolution in bacterial endosymbionts having extreme base compositions. Mol Biol Evol 16:1586-1598

8. Cordaux R, Michel-Salzat A, Bouchon D (2001) Wolbachia infection in crustaceans: novel hosts and potential routes for horizontal transmission. J Evol Biol 14:237-243

9. Covacin C, Barker SC (2006) Supergroup F Wolbachia bacteria parasitise lice (Insecta: Phthiraptera). Parasitol Res 100:479-485

10. Floyd RM, Rogers AD, Lambshead PJD, Smith CR (2005) Nematode-specific PCR primers for the 18S small subunit rRNA gene. Mol Ecol Notes 5:611-612

11. Gotoh T, Noda H, Hong XY (2003) Wolbachia distribution and cytoplasmic incompatibility based on a survey of 42 spider mite species (Acari: Tetranychidae) in Japan. Heredity 91:208-216

12. Hartelt K, Oehme R, Frank H, Brockmann SO, Hassler D, Kimmig P (2004) Pathogens and symbionts in ticks: prevalence of Anaplasma phagocytophilum (Ehrlichia sp.), Wolbachia sp., Rickettsia sp., and Babesia sp. in Southern Germany. Int J Med Microbiol 37:86-92

13. Lo N, Evans TA (2007) Phylogenetic diversity of the intracellular symbiont Wolbachia in termites. Mol Phylogenet Evol 44:461466

14. Martin D, Williamson C, Posada D (2005) RDP2: recombination detection and analysis from sequence alignments. Bioinformatics 21:260-262 
15. Miller WJ, Riegler M (2006) Evolutionary dynamics of wAu-like Wolbachia variants in neotropical Drosophila spp. Appl Environ Microbiol 72:826-835

16. O'Neill SL, Giordano R, Colbert AM, Karr TL, Robertson HM (1992) 16S rRNA phylogenetic analysis of the bacterial endosymbionts associated with cytoplasmic incompatibility in insects. Proc Natl Acad Sci U S A 89:2699-2702

17. Panaram K, Marshall JL (2006) F supergroup Wolbachia in bush crickets: what do patterns of sequence variation reveal about this supergroup and horizontal transfer between nematodes and arthropods? Genetica 130:53-60

18. Posada D, Crandall KA (1998) Modeltest: testing the model of DNA substitution. Bioinformatics 14:817-818

19. Prendini L (2001) A review of synonyms and subspecies in the genus Opistophthalmus C.L. Koch (Scorpiones: Scorpionidae). African Entomol 9:17-48

20. Prendini L (2001) Substratum specialization and speciation in southern African scorpions: the Effect Hypothesis revisited. In: Fet V, Selden PA (eds) Scorpions 2001. In Memoriam Gary A Polis. Burnham Beeches, Bucks, UK: British Arachnological Society, pp 113-138

21. Prendini L (2005) Scorpion diversity and distribution in southern Africa: pattern and process. In: Huber BA, Sinclair BJ, Lampe KH (eds) African biodiversity: molecules, organisms, ecosystems. Proceedings of the 5th International Symposium on
Tropical Biology, Museum Alexander Koenig, Bonn. New York: Springer Verlag, pp 25-68

22. Rowley SM, Raven RJ, McGraw EA (2004) Wolbachia pipientis in Australian spiders. Curr Microbiol 49:208-214

23. Rozas J, Sanchez-DelBarrio JC, Messeguer X, Rozas R (2003) DnaSP, DNA polymorphism analyses by the coalescent and other methods. Bioinformatics 19:2496-2497

24. Sakamoto JM, Feinstein J, Rasgon JL (2006) Wolbachia infections in the Cimicidae: museum specimens as an untapped resource for endosymbiont surveys. Appl Environ Microbiol 72:3161-3167

25. Stouthamer R, Breeuwer JAJ, Hurst GD (1999) Wolbachia pipientis: microbial manipulator of arthropod reproduction. Ann Rev Microbiol 53:71-102

26. Swofford DL (2002) PAUP*: phylogenetic analysis using parsimony (* and other methods), version $4.0 \mathrm{~b}$ 10. Sunderland, Massachusetts: Sinauer Associates

27. Werren JH (1997) Biology of Wolbachia. Annu Rev Entomol 42:587-609

28. Werren JH, Windsor DM (2000) Wolbachia infection frequencies in insects: evidence of a global equilibrium? Proc R Soc Lond B Sci 267:1277-1285

29. Zeh DW, Zeh JA, Bonilla MM (2005) Wolbachia, sex ratio bias and apparent male killing in the 16 harlequin beetle riding pseudoscorpion. Heredity 95:41-49 